\title{
Innenraumallergene und Carpet Crawlers
}

\section{Indoor Allergens and Carpet Crawlers}

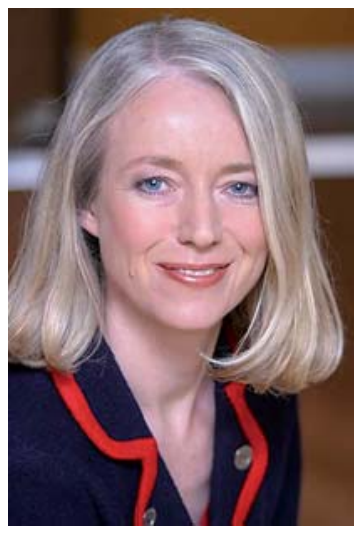

Prof. Dr. Christiane Bayerl
Bibliografie

DOI http://dx.doi.org/

10.1055/s-0030-1255908

Akt Dermatol 2010; 36; 397

(c) Georg Thieme Verlag KG .

Stuttgart · New York

ISSN 0340-2541

Korrespondenzadresse

Prof. Dr. med. Christiane BayerI Klinik für Dermatologie und Allergologie Wiesbaden HSK, Wilhelm-Fresenius-Klinik Städtisches Lehrkrankenhaus der Universität Mainz

Aukammallee 39

65191 Wiesbaden

christiane.bayerl@

hsk-wiesbaden.de
Der Winter hat begonnen und die Heizungen wurden angedreht. Da wird eine Menge Staub aufgewirbelt. Hausstaub ist keine tote Materie, da krabbelt und wächst so alles Mögliche darin. Die Leitlinien zur Allergieprävention zeigen eine neue Wertung der Innenraumallergene. Die alte Regel, dass Hausstaubmilben im Umfeld eines Babys mit atopischer Familienanamnese saniert werden müssen, geriet ins Wanken. Zumindest, was die primäre Prävention anbelangt, wurde diese Empfehlung verlassen, gilt aber noch für die sekundäre oder tertiäre Prävention. Es wird aber weiterhin empfohlen, das Innenraumklima so zu gestalten, dass kein Pilzwachstum stattfinden kann. Eine hohe Luftfeuchtigkeit ist zu vermeiden. Lüften wird empfohlen. Zu den Innenraumallergenen zählen aber auch die volatilen organischen Verbindungen, Formaldehyd aus neuen Möbeln oder Farben, die bei der Renovierung eingesetzt wurden. Diese aerogene Exposition sollte limitiert werden.

Ein Sammelplatz für Innenraumallergene ist neben dem Altbekannten, der Matratze und dem Badvorleger - also den Bereichen, in denen viele menschliche Schuppen fallen, schon immer auch ein Teppich. Der Liebhaber alter farbschöner Teppiche wäre froh, wenn diese Wohnaccessoires irgendwie „entschuldet“ werden könnten. Krabbelt da wirklich so viel auf dem Teppich? Bekannt ist, dass auch Pestizide in Teppichen und im Hausstaub gefunden werden, aber das scheint die Besiedler eines Teppichs nicht zu stören.

Abhilfe kann geschaffen werden. Aus Studien zur Reduktion von Bleiexposition sind bestimmte Staubsauger bekannt, die auch effektiv Allergene aufnehmen können. Dazu notwendig ist ein sogenannter HEPA (high efficiency particulate air)-Filter. Eine Trockendampfreinigung eines Teppichs war im Vergleich nicht effektiver. Es wurde nachgewiesen, dass sowohl die Konzentrationen von polyzyklischen aromatischen Hydrokarbonen als auch die der Hausstaubmilben so um mehr als 50\% reduziert werden können [1].

Die Pilzsporen im Innenraum spiegeln die Besiedlung im Freien wider. Das Maximum des Sporenfluges von Alternaria, Cladosporium und Penicillium liegt im August in Mutter Natur und hat im Innenraum einen Peak zu Beginn der Heizsaison. Raumkühler erhöhen bei Patienten mit Asthma und allergischer Rhinitis das Risiko auf Schimmelpilze im Hauttest zu reagieren. Patienten mit Zentralheizung reagierten zu 19\% auf Schimmelpilze im Hauttest gegenüber $42 \%$ der Patienten, deren Klima- tisierung über einen Raumkühler erfolgte - analog waren die Ergebnisse für die Hausstaubmilbentestung [2]. Obwohl eine Sensibilisierung auf Pilzallergene auch bei Stadtkindern (nicht nur auf dem Bauernhof) vorkommt, war der Zusammenhang zur Morbidität nicht klar. In einer Studie, die über 2 Jahre lief, wurde die Korrelation der Symptomatik bei Kindern mit positivem Hauttest mit der Konzentration der gemessenen Sporen untersucht. Die Effekte auf die Asthma-Morbidität bei Kindern waren abhängig von der Pilzkonzentration allgemein im Freien und im Innenraum speziell von der Konzentration von Penicillium-Spezies [3]. Es macht wohl doch Sinn, diese Allergene bei Sensibilisierten zu meiden, aber das ist dann auch keine primäre Prävention mehr.

Die „Carpet Crawlers“ ist eine Single von Genesis aus dem Jahr 1975. Der Titel bezieht sich dabei nicht auf die Hausstaubmilben oder die Kakerlaken. Der Held der Single findet sich auf einem roten Teppich wieder, der von knieenden Menschen bedeckt ist und auf eine rote Tür am Ende der Halle zuführt. Die Hauptperson kann sich aber durch diese knieenden Menschen frei hindurchbewegen und gelangt zu einem Tisch, der mit Kerzen dekoriert und mit Speisen gedeckt ist. Dahinter führt eine Wendeltreppe aus dem Sichtfeld des Betrachters hinaus. Die Lyrik des Textes mag interpretiert werden, wie es die Fantasie erlaubt, hat aber nichts mit Krabbeltieren zu tun.

Wohin die allergologische Wendeltreppe führt, ist auch nicht zu sehen. Ich kann aber aus der allergologischen Weiterbildungspalette für 2011 schon einen Termin für Ihren Kalender absehen. Am 11. und 12. 2. 2011 findet in Wiesbaden in Kurhaus erstmals das Allergo-Update statt - und da werden Sie sehen, wo es hingeht mit der Allergologie.

Ihre

Christiane Bayerl

\section{Literatur}

$1 \mathrm{Yu} C$, Yiin $L M$ et al. Evaluation of HEPA vacuum cleaning and dry steam reducing levels of polycylic aromatic hydrocarbons and dust mite allergens in carpets. J Environ Monit 2009; 11: 205-211

2 Prasad C, Hogan MB et al. Effect of evaporative coolers on skin test reactivity to dust and molds in a desert environment. Allergy Asthma Proc 2009; 30: 624-627

3 Pongracic JA, O'Connor GT et al. Differential effects of outdoor versus indoor fungal spores on asthma morbidity in inner-city children. J All Clin Immunol 2009; 125: $593-599$ 